\title{
The First Steps in the Life of a GRB
}

\author{
Miguel A. Aloy \\ Max-Planck-Institut für Astrophysik, Karl-Schwarzschild-Str. 1, 85741 Garching, \\ Germany \\ maa@mpa-garching.mpg . de
}

\begin{abstract}
Summary. We present some preliminary results of relativistic hydrodynamic simulations of post-neutron star merger disks as potential candidates for progenitors of short-lasting gamma-ray bursts. We discuss some of the generic conditions under which a gamma-ray burst can be initiated in this kind of progenitor and the main characteristics of the resulting outflow.
\end{abstract}

\section{Introduction}

Gamma-ray bursts (GRBs) are sudden releases of energy which are, most probably, linked with catastrophic collapse events of massive stars (collapsars [17]; hypernovae, [13]) or with mergers of compact binaries $[5,6,11,12]$. In all these models a fraction of the gravitational binding energy released by accretion of matter from a thick torus girding a stellar mass black hole (BH) is thought to power a pair fireball. Assuming that the baryon load of the fireball is not too large, the baryons are accelerated together with the $\mathrm{e}^{+} \mathrm{e}^{-}$ pairs to Lorentz factors $>10^{2}$ [4].

Due to their different duration and spectral properties GRBs are commonly divided in two classes: short $(\leq 2 \mathrm{~s})$ and long $(\geq 2 \mathrm{~s})$ GRBs [9]. The long subtype of GRBs has been quite extensively observed and there is a relatively large number of afterglow multi band observations from radio to $\mathrm{X}$-rays. These observations have shed some light on the kind of progenitors and environments in which such progenitors reside. From the numerical point of view, the generation and early evolution of long GRBs in the framework of the collapsar model has been studied using 2D Newtonian [10] and relativistic hydrodynamics $[2,3,18]$. Observations of short GRBs are less numerous and it has not been possible to detect them in multi frequency searches.

The scenario arising after the merging process of a compact binary system consists of a central $\mathrm{BH}$ of a few solar masses girded by a thick accretion torus whose mass is of $0.05-0.3 \mathrm{M}_{\odot}[14,8]$. Once the thick disk is formed, up to $\sim 10^{51}$ erg can be released above the poles of the $\mathrm{BH}$ in a region that contains less than $10^{-5} \mathrm{M}_{\odot}$ of baryonic matter due to the release of energy via $\nu-\bar{\nu}$ annihilations. In principle, this may lead to the acceleration of this matter to ultrarelativistic speeds accounting for a successful GRB. If the duration of the event is related to the lifetime of the system [15] this kind of events can 
only belong to the class of short GRBs because the expected time scale on which the $\mathrm{BH}$ engulfs the disk is fractions of a second [14].

In this work we address the question of whether a local deposition of energy around the remnant left over from the merger of two neutron stars can yield the formation of a pair of relativistic, collimated plasma outflows in opposite directions that can account for short GRBs. We employ 2D general relativistic hydrodynamic numerical simulations to study the properties of the outflows generated when pure thermal energy is released in a wide angle cone around the rotation axis of the system consisting of a stellar mass BH surrounded by a thick accretion torus. We use the high-resolution shock-capturing code GENESIS [1] to integrate the general relativistic hydrodynamic equations in $2.5 \mathrm{D}$ (i.e., in spherical $(r, \theta)$ coordinates assuming that there is no $\phi$ dependence of any physical quantity although the $\phi$ components of any vector can have non-zero values). Among the issues that we want to study are: the viability of the scenario of compact object mergers for producing ultrarelativistic outflows: the mechanism that can account for collimation (if any) of the outflowing plasma; the expected duration of GRB events generated in this framework; and the relation to the time during which the source of energy is active.

\section{Initial Model and Numerical Set Up}

We have constructed two initial models in which the gravitational field is provided by a Schwarzschild $\mathrm{BH}$ of $3 \mathrm{M}_{\odot}$ (models type-A) and $2.44 \mathrm{M}_{\odot}$ (models type-B) located at the center of the system (effects on the dynamics due to the self-gravity of the accretion torus or the external environment are neglected). These black holes are surrounded by thick accretion disks for which the initial configurations are built either by guidance through the data of Ruffert \& Janka [14] and then letting the model relax until a torus of $0.1 \mathrm{M}_{\odot}$ is obtained (type-A), or by analytically following a prescription very. close to that of Font \& Daigne [7] in order to build an equilibrium torus of $0.07 \mathrm{M}_{\odot}$ around a Schwarzschild BH (type-B). The initial models include an environment which is of high density and non uniform in type-A models. In type-B models it is spherically symmetric, with low density which decreases with radius $\left(\rho \sim r^{-3.4}\right)$ and that has a total mass of $2.52 \times 10^{-7} \mathrm{M}_{\odot}$. These initial configurations, although they are ad hoc (i.e., not the result of a full computation of the merger epoch), mimic the expected state of a remnant of the merger of a compact binary system sufficiently well for our purposes. We assume equatorial symmetry, and we cover $90^{\circ}$ in the angular $\theta$-direction with 200 uniform zones. In the $r$-direction the computational grid consists of 400 (type-A) or 500 (type-B) zones spaced logarithmically between the inner boundary and an outermost radius of $R_{\max }=2 \times 10^{10} \mathrm{~cm}$ (thus, we can study the evolution of any outflow up to $\sim 0.5 \mathrm{~s}$ ). The equation of state includes the contributions of non-relativistic nucleons treated as a mixture 
of Boltzmann gases, radiation, and an approximate correction due to $e^{+} e^{-}$ pairs [16]. Complete ionization is assumed, and the effects due to degeneracy are neglected.

In a consistent post neutron star merger model an outflow will be powered by any process which gives rise to a local deposition of energy and/or momentum, as e.g., $\nu \bar{\nu}$-annihilation, or magneto-hydrodynamic processes. We mimic such a process by releasing pure thermal energy in a prescribed cone around the rotational axis of our system. In the radial direction the deposition region extends from the inner grid boundary located at 2 gravitational radii $\left(R_{g}=G M / c^{2} ; G, M\right.$ and $c$ being the gravitational constant, the mass of the $\mathrm{BH}$ and the speed of light in vacuum, respectively) to the outer radial boundary. In the angular direction, the opening half-angle of the deposition cone $\left(\theta_{0}\right)$ was chosen to be in the range $30^{\circ}-75^{\circ}$. From the annihilation rate distribution computed in [14] and [8], we infer a power law distribution for the energy deposited per unit of volume in the surrounding of the system whose explicit form was approximated as $\dot{q}=\dot{q}_{0} z^{-n}$, where $z$ is the distance along the rotation axis, $n$ is the power law index ( $n=5$ hereafter) and $\dot{q}_{0}$ is a normalization factor that we use to fix the total energy deposition rate $(\dot{E})$.

\section{Results}

We have done a parameter study addressing three different aspects of the morphology and dynamics of outflows resulting from neutron star merger remnants. The first one is the question for the consequences of increasing $\dot{E}$ from $5 \times 10^{49} \mathrm{ergs}^{-1}$ to $5 \times 10^{51} \mathrm{erg} \mathrm{s}^{-1}$, using a fixed value of the opening half-angle $\left(\theta_{0}\right)$ of the deposition region. The second aspect is the dependence of the results when varying $\theta_{0}$ while keeping the rest of the parameters fixed. Finally, we compare models having the same rate of energy deposition per unit of volume.

For energy deposition rates larger than a certain threshold $\dot{E}_{\mathrm{th}}$, all the models lead to either relativistic jets or ultrarelativistic winds (i.e., fireballs). The threshold is due to the need of overcoming the ram pressure $p_{\text {ram }}$ that is exerted by the infalling external medium onto the new born fireball close to its initiation site. If the amount of energy per unit of volume pumped into the deposition region (in an interval of about of the free falling time of the fluid located at distances of the order of the radius of the torus) is not larger than $p_{\text {ram }}$, the fireball is swallowed by the $\mathrm{BH}$. The precise value of the threshold is model dependent as long as the densities and accretion velocities outside the thick torus depend on the details of the merger phase. For type-A models we find $\dot{E}_{\mathrm{th}} \sim 10^{49} \mathrm{erg} \mathrm{s}^{-1}$, while for type-B models $\dot{E}_{\mathrm{th}} \lesssim 10^{48} \mathrm{erg} \mathrm{s}^{-1}$. The smaller value in type-B models is due to their smaller ambient density.

Depending on the energy deposition rate and on the ambient density we find that the outflows are either jets (i.e., outflows where the lateral boundaries are causally connected) having a very small opening angle $\left(\lesssim 8^{\circ}\right)$ or 
relatively wide opening angle $\left(\lesssim 25^{\circ}\right)$ winds (i.e., the lateral boundaries are not causally connected). Models close to the thresholds of the energy deposition rate or with a high density environment tend to form relativistic $(\Gamma \sim 10)$, low density, knotty jets whose head propagates at mildly relativistic speeds $(\sim 0.6 c)$. In contrast, models well above the threshold with dense environments or, independent of the deposition rate, in case of diluted environments either tend to form conical, ultrarelativistic $(\Gamma \gtrsim 400)$ winds which are smooth, propagate at relativistic speeds $(\sim 0.97 c)$ and can be fitted by analytic power laws in case of models of type-A, or they propagate at ultrarelativistic speeds ( $\gtrsim 0.9999 c$ ) being rather irregular due to the effect of large Kelvin-Helmholtz $(\mathrm{KH})$ instabilities originating from their interaction either with the torus, or with the environment in case of type-B. Indeed, the growth of $\mathrm{KH}$ modes determines whether the profiles of the physical variables are smooth and monotonically decreasing in the $r$-direction (type-A), or non-smooth and non monotonic (type-B). The larger growth of surface instabilities in models of type- $\mathrm{B}$ is due to the larger density contrast with respect to the environment in these type of models. An effect of the $\mathrm{KH}$ instabilities is to entrain mass into the relativistic outflows of type-B models. The amount of entrained mass is comparable with that of models of type-A. However, in type-B models, the amount of matter piled up ahead of the outflow is much smaller (because there is much less mass in the ambient) leading to a highly relativistic propagation velocity of the fireballs of type-B models, while only allowing for mildly relativistic speeds in type-A models. Indeed, the speed of propagation of the fireball is so large that models of type-B will lead to successful GRBs (in contrast to models of type-A; see below).

Increasing the energy deposition rate yields, independently of the model, an increase of the average Lorentz factor, and a decrease of the average density of the outflow at any given time. In models of type-A, the increase of $\dot{E}$ results in a transition in the outflow morphology from relativistic jets $\left(\dot{E}<10^{51} \mathrm{erg} \mathrm{s}^{-1}\right)$ to ultrarelativistic wind-like outflows $\left(\dot{E}>10^{51} \mathrm{erg} \mathrm{s}^{-1}\right)$. In models of type- $B$, we find ultrarelativistic winds for all energy deposition rates considered $\left(\dot{E}>5 \times 10^{48} \mathrm{erg} \mathrm{s}^{-1}\right)$. For energy deposition rates leading to conical wind structures, the opening angle of the outflow $\left(\theta_{w}\right)$ is quite insensitive to the exact value of $\dot{E}$ (although it slightly increases with increasing $\dot{E})$, its value being $\sim 20^{\circ}-30^{\circ}$.

In type-A models, decreasing $\theta_{0}$ produces a transition from narrow jets to wide angle winds. In type- $\mathrm{B}$ models there is almost no difference in the opening angle of the wind when we vary $\theta_{0}$ between $30^{\circ}$ and $75^{\circ}$. However, there is a slight decrease of $\theta_{w}$ and an increase of the mass entrained (because of the development of KH instabilities) with increasing $\theta_{0}$. In general, increasing $\theta_{0}$ while keeping $\dot{E}$ constant leads to smaller average Lorentz factors in the resulting outflow.

Releasing the same total energy per unit of volume and time leads in models of both types to a larger baryon loading, when both $\theta_{0}$ and $\dot{E}$ are 
increased. In type-A models this also implies that there is a trend to form relativistic jets instead of winds, when we increase $\theta_{0}$. In type- $B$ models we always form winds but they have more baryon loading with increasing $\theta_{0}$ and $\dot{E}$. The reason for this is that although the total energy deposition per unit of volume is the same, the model having the smallest deposition angle sweeps up less ambient mass (and drags less mass from the torus) than the model having the largest deposition angle and energy deposition rate.

We conclude that the opening angle of the resulting outflow, provided it is a jet, is set by the environmental conditions (mainly, the density). If the generated outflow is a wind, then the complete collimation process happens in less than $1 \mathrm{~ms}$ (approximately, the light crossing time of the torus) and $\theta_{w}$ is set by the opening angle of the torus, and neither by the external medium (which has much less inertia in type-B models because it is much more rarefied), nor by the angular size of the deposition region.

We have checked the evolution of two models after the shut down of the energy deposition. It turns out that outflows propagating in high density environments (type-A) will not yield successful GRBs while models with diluted environments (type-B) can do so. The reason being that, in type-B models, the speed of propagation of the leading radial edge of the fireball is faster than the rear one when the energy deposition is shut down. This is not the case in type-A models because they sweep up more ambient mass and the leading front of the outflow slows down. We find that in type-B models the fireball even stretches substantially in radial direction, because the propagation velocity of its leading front is larger than its rear edge. This points to the possibility that the duration of the GRB emission can be much larger than the time of the activity (i.e., of release of energy) of the central source.

\section{References}

1. M.A. Aloy, J.M. Ibáñez, J.M. Martí, E. Müller: Astrophys. J. Suppl. 122, 151 (1999)

2. M.A. Aloy, E. Müller, J.M. Ibáñez, J.M. Martí, A.I. MacFadyen: Astrophys. J. Lett. 531, L119 (2000)

3. M.A. Aloy, J.M. Ibáñez, J.A. Miralles, V. Urpin: Astron. Astrophys. 396, 693 (2002)

4. G. Cavallo, M.J. Rees: Mon. Not. R. Astron. Soc. 183, 359 (1978)

5. D. Eichler, M. Livio, T. Piran, D.N. Schramm: Nature 340, 126 (1989)

6. J. Goodman: Astrophys. J. Lett. 308, L47 (1986)

7. J.A. Font, F. Daigne: Mon. Not. R. Astron. Soc. 334, 383 (2002)

8. H.-Th. Janka, Th. Eberl, M. Ruffert, C.L. Fryer: Astrophys. J. Lett. 527, L39 (1999)

9. C. Kouveliotou et al. : Astrophys. J. Lett. 413, L101 (1993)

10. A.I. MacFadyen, S.E. Woosley: Astrophys. J. 524, 262 (1999)

11. R. Mochkovitch, M. Hernanz, J. Isern, X. Martin: Nature 361, 236 (1993)

12. B. Pacyński: Astrophys. J. Lett. 308, L43 (1986) 
13. B. Pacyński: Astrophys. J. 494, L45 (1998)

14. M. Ruffert, H.-Th. Janka: Astron. Astrophys. 344, 573 (1999)

15. R. Sari, T. Piran: Astrophys. J. 485, 270 (1997)

16. J. Witti, H.-Th. Janka, K. Takahashi: Astron. Astrophys. 286, 841 (1994)

17. S.E. Woosley: Astrophys. J. 405, 273 (1993)

18. W. Zhang, S.E. Woosley, A.I. MacFadyen: Astrophys. J. 586, 356 (2003) 


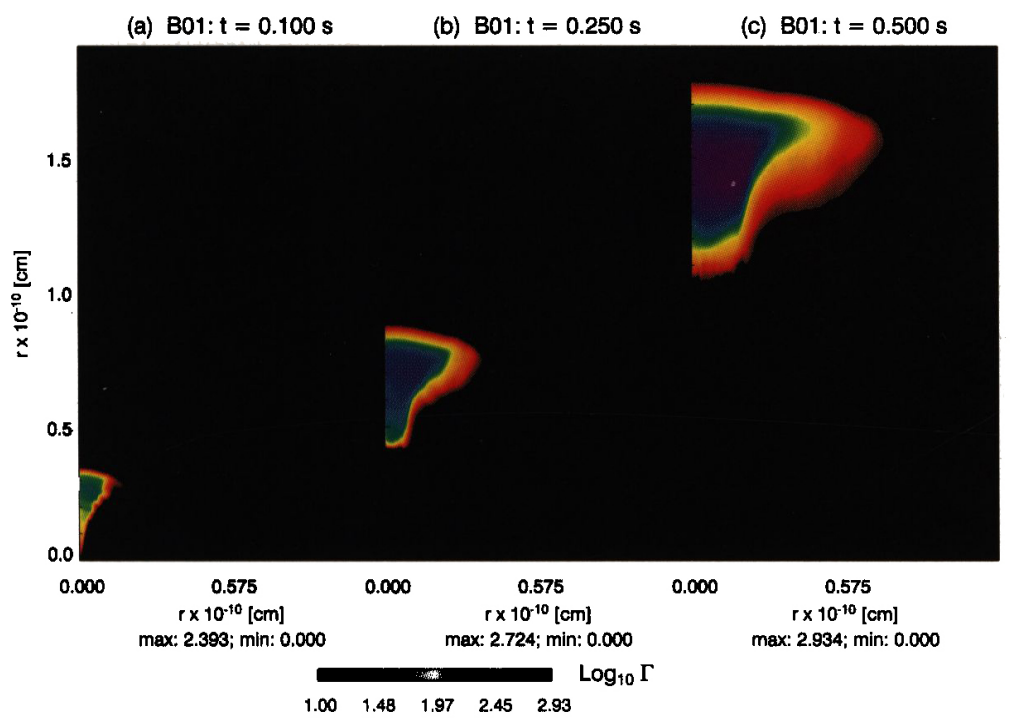

Evolution of the Lorentz factor (in logarithmic scale) of the outflow after the central energy release shut-off The time of each snapshot is printed on top of each panel. The scale is limited to enhance the variations in the ultra-relativistic core of the outflow. The values of the minimum logarithm of the Lorentz factor a at each time are shown below each panel. In this model, thermal energy has been released during $100 \mathrm{~ms}$ at a rate of $2 \cdot 10 \% \mathrm{erg} / \mathrm{s}$ in a conical region around the symmetry axis of half-opening angle $45 \mathrm{deg}$ in the vicinity of a $2.44 \mathrm{M}_{\odot}$ black hole. Comparing the middle and right panels with the left one, it is noticeable

the radial stretching of the ultrarelativistic core of the fireball. A possible consequence of this stretching is that the duration of the GRB event can be larger than the time of activity of the source. At the final computed time $(0.5 s)$ the Lorentz factor of the fireball is still increasing and does not show any sign of saturation yet.

Plate (Aloy)

\section{Plate 5.}

